\title{
Vaccine evaluation: lessons from a meningococcal $B$ vaccine
}

\author{
Avishay Sarfatti, ${ }^{1}$ Federico Martinón-Torres, ${ }^{2}$ Simon Nadel ${ }^{3}$
}

Vaccination is among the most effective public health interventions of the past century, with immunisations against some of the most common and devastating childhood infections already in use to great effect. The development of new vaccines depends on advances in the field of vaccinology that carry high monetary costs. In most countries, these costs pose an obstacle to adoption of new vaccines. To that end, together with the scientific evaluation of new vaccines, an economic evaluation is vital. This aims to determine whether investment in the proposed vaccine is worthwhile.

In this article, we will examine one such case study: the evaluation of a new meningococcal B (MenB) vaccine in the UK by the Joint Committee on Vaccination and Immunization (JCVI). We are well aware of the constraints imposed by a definite health budget; at the same time, we would like to raise awareness to the problems inherent in and the somewhat speculative nature of the economic model when applied to vaccine evaluation. Though some of the aspects of this case are specific to the UK, the principles of this evaluation are relevant to all modern health systems.

In March 2014, JCVI made public its decision to support the introduction of a MenB vaccine (Bexsero) that had been licensed for use in Europe the year before. $^{1}{ }^{2}$ JCVI recommended that the vaccine should be introduced into the UK routine infant immunisation schedule if the appropriate price per vaccine dose could be negotiated.

This decision is welcome news to the medical profession, to meningitis patients' advocacy groups, to the population in general and to the vaccine manufacturers. The process by which Bexsero was recommended highlighted the central role that

\footnotetext{
${ }^{1}$ Paediatric Intensive Care, Paediatric Intensive Care Unit, St. Mary's Hospital, London, UK; ${ }^{2}$ Translational Pediatrics and Infectious Diseases, Hospital Clínico Universitario de Santiago de Compostela, Santiago de Compostela, Spain; ${ }^{3}$ Paediatric Intensive Care, Paediatric Intensive Care Unit, St. Mary's Hospital and Imperial College London, London, UK

Correspondence to Dr Simon Nadel, Paediatric Intensive Care, Paediatric Intensive Care Unit, St. Mary's Hospital and Imperial College London, Praed Street, London W21NY, UK;

nadel@imperial.ac.uk
}

health economics and cost-effectiveness analysis play in such decisions. The difficulties that JCVI faced when reaching its eventual decision suggest that it is now time to critically examine the methods and models used by JCVI.

JCVI is an independent expert committee that holds an advisory role to the UK Department of Health and the Secretary of State for Health. ${ }^{3}$ JCVI is a professional body and not a political one. It does not make policies in its own right and has no regulatory function. Before making recommendations whether or not to recommend a vaccine, JCVI considers evidence on disease burden, vaccine safety and efficacy and on the impact and cost effectiveness of possible immunisation strategies. Similar Immunization Technical Advisory Groups (ITAGs) exist in many other countries, including the USA, Canada, Australia, Spain and Israel to name but a few. ${ }^{4}$

Given the reality of a finite health budget, and that some health interventions need to be chosen at the expense of others, ITAGs are often asked to perform a cost-effectiveness analysis as part of vaccine evaluation. In the UK, in order to assess whether a vaccination programme can be considered cost effective, JCVI has adopted methodology developed by the National Institute for Health and Care Excellence (NICE). A vaccination programme can be considered cost effective if its health benefits (both the direct benefits to those vaccinated and the indirect benefits to the unvaccinated population) meet an agreed monetary threshold of cost per quality-adjusted life-year (QALY) gained.

In order to conduct a cost-effectiveness calculation, JCVI needs to ascertain the cost of implementing a new immunisation programme, the money likely to be saved as a direct and indirect consequence of disease prevention, the number of cases averted and thereby the number of QALYs gained. A threshold of $£ 20-30000$ per QALY gained is the cost under which an intervention is considered to be cost effective and is therefore recommended.

A full discussion of the problems this methodology presents is beyond the scope of this paper. Here we will discuss the reasons why we find this calculation to be inadequate when considering introduction of a new vaccine and will demonstrate why the value of preventing a disease by immunisation cannot be fully assessed through the calculations mentioned above. We will use the example of Bexsero to demonstrate this.

\section{THE EXAMPLE OF BEXSERO IN THE UK}

The cost-effectiveness evaluation of Bexsero was initially based on an independent economic study conducted by the University of Bristol and the London School of Hygiene and Tropical Medicine. ${ }^{5}$ That study found Bexsero to be cost effective across a range of vaccine costs. This model was subsequently adjusted to take into account advice from JCVI's Meningococcal Subcommittee, an adjustment that was followed by JCVI's interim statement. ${ }^{6}$ JCVI announced in that statement that Bexsero would not be cost effective at any vaccine price. Effectively, even if the vaccine were free, its introduction could not be cost effective.

There followed an unprecedented and progressive consultation process during which JCVI took note of opinions from various stakeholders including patient advocate groups, the pharmaceutical industry and others. The financial model was revised again to include additional parameters, such as litigation costs to the National Health Service (NHS) associated with MenB disease and the loss of quality of life to family members of cases with long-term sequelae from the disease. ${ }^{7}$ Some of the inputs to the original model were altered, among them the incidence of invasive meningococcal disease being represented by an average incidence over a longer time period, rather than just the previous two years' figures, which were unusually low; and an evidence-based adjustment of the possible effect of the vaccine on nasopharyngeal carriage. In addition, JCVI took into account new evidence that had recently become available. These revised inputs to the financial model subsequently allowed JCVI to recommend introduction of the Bexsero vaccine if the appropriate price could be negotiated with the manufacturer. ${ }^{2}$

This revision of JCVI's recommendation highlights how dependent this type of evaluation is on a series of assumptions. When constructing such cost-effectiveness models, three key areas are examined:

1. the effects of the vaccine on disease burden

2. the monetary cost of the disease to society

3. the cost of implementing an immunisation programme.

The latter can be estimated relatively accurately in a well-developed health 
system such as the UK, and we will not address it here.

To analyse the effect of the vaccine on disease burden requires JCVI to make assumptions about disease prevalence, vaccine efficacy, duration of protection offered by the vaccine, strain coverage and effects on carriage and herd immunity. Much of this information is usually available only after widespread vaccine implementation. This has been illustrated following the introduction of other vaccines, such as the meningococcal $\mathrm{C}$ (MenC) and pneumococcal conjugate vaccines. ${ }^{89}$ In this respect, it is interesting to note that the three costeffectiveness evaluations for Bexsero done to date, from the Netherlands, Canada and the UK, made different assumptions about vaccine efficacy, duration of protection and effect on carriage and therefore on likely herd immunity. ${ }^{5} 1011$ Possible effects on carriage played a prominent role in the discussions about the cost effectiveness of Bexsero. The different models used a variety of figures for the influence on carriage, ranging from $0 \%$ to $30 \% .^{2}$

It is worth noting that a cost-effectiveness calculation undertaken before the introduction of the MenC vaccine found this vaccine to be cost effective at just under $£ 30000$ per QALY. That this was the case was due to several incorrect assumptions made in the analysis: first, there was an assumption that the vaccine would offer lifelong immunity; it is now clear that immunity from the MenC vaccination wanes over time, with efficacy falling from $95.9 \%$ after the primary schedule to $30.7 \%$ within 12 months. ${ }^{12}$ Had this been known when JCVI evaluated the MenC vaccine in 1998, the MenC vaccine would have been deemed not cost effective. Second, the costeffectiveness calculations done at that time ignored the now recognised effects on carriage and therefore herd immunity. ${ }^{13} \mathrm{We}$ therefore owe the successful introduction of the MenC vaccine to incorrect assumptions balancing each other out.

Another illustration of the limits of preimplementation cost-effectiveness calculations involves pneumococcal conjugate vaccine. Before it was introduced into the USA, its cost effectiveness was estimated to be US\$80 000-176000 per QALY. These figures would prohibit its use in the UK. A repeat analysis that used the 5-year experience following vaccine introduction incorporated the effect of herd immunity, and thus reduced the cost to US\$7500 per QALY. $^{9}$

Another area of importance in costeffectiveness evaluations is the cost of the disease to health services, and more broadly to society. This is even more difficult to determine. The costs of treating a child affected by a life-threatening disease with long-term sequelae, both in the short term and for many years to follow, cannot be accurately market priced. A recent estimate found that the health-related cost to NHS of managing a child with meningococcal disease with major sequelae is $£ 131670$ 151651 in the acute phase alone. ${ }^{14}$ This study found that the lifelong undiscounted medical cost to NHS of looking after a child with major sequelae of meningococcal disease is $£ 612352-952076$, increasing to $£ 3053694-3769579$ if costs to society are included. These cost estimates assessed the cost implications of two specific scenarios (severe meningococcal septicaemia and severe meningococcal meningitis with neurological sequelae). It is striking to read the many assumptions the authors had to make in order to reach these specific costs. It is clear that even small variations in these assumptions would have a significant impact on the overall cost.

What would be the financial cost of a child with a milder acute illness, who as a result fails to achieve his or her full cognitive and physical potential? Behind this question is a socioeconomic concept of outcome-related productivity gains. It argues that allowing children to achieve their full cognitive and physical potential would provide any given society with financial gains.

A study comparing survivors of bacterial meningitis 12 years after the acute infection, with their peers, found substantial excess risk of intellectual, cognitive and auditory impairment, which resulted in lower educational achievement and higher risk of behavioural disorders. ${ }^{15}$ Clearly, assigning an agreed monetary value to these possible gains to society is a difficult task.

\section{OTHER EFFECTS}

It is important to emphasise that economic evaluation using methodology designed for curative treatments significantly disadvantages the cost-effectiveness analyses of preventative therapies such as vaccination. This is so not only for the reasons mentioned thus far but also because cost-effectiveness analysis does not account for the additional added value offered by vaccination such as the advantages of primary prevention, the consequent reduction in use of antimicrobials and therefore effects on antimicrobial resistance, the possibility of disease eradication and the general improvement in childhood health.

The pharmaceutical industry is a central part of the pipeline for vaccine development by providing vital funding and resources for research and development. The current financial model for evaluation may discourage research by the pharmaceutical industry and academia to develop novel vaccines and technologies. Developments such as reverse vaccinology, structural vaccinology and novel adjuvants have great potential to advance vaccine development, perhaps beyond the prevention of infectious diseases. $^{16}$

\section{CONCLUSION}

We acknowledge that in the reality of a finite health budget a process needs to be in place to prioritise some health interventions over others. The current methodology to assess cost effectiveness of medical interventions is widely used by NICE in the UK and similar bodies internationally, and is likely to continue to be so until a more robust method is available. At the same time, it is important to recognise the deficiencies of the current methodology when used to assess new vaccines; that it is dependent on speculative parameters inserted into the cost-effectiveness model; and that it is inherently disadvantageous to the introduction of new vaccines. The UK experience with Bexsero illustrates how unsound economic considerations may overshadow any potential benefits to individuals and society of a novel disease prevention programme, that is, through the introduction of a new immunisation.

The news that JCVI is currently setting up a working group on cost effectiveness is to be welcomed. ${ }^{17}$ We suggest that efforts should be made to separate the financial from the medical evaluation of vaccines. Evaluation of new vaccines should involve two clearly distinct processes: the first would be a medical/professional opinion regarding the medical need for the vaccine, its efficacy and its safety. The second should consider the cost effectiveness of the vaccine. However, the latter should acknowledge that unfortunately there are no infallible tools to fully evaluate vaccine cost effectiveness at present. To suggest that the current methods of vaccine evaluation assist an accurate decision-making process is misleading for the reasons specified above. We suggest that health economics should guide, rather than determine, immunisation policy.

We congratulate JCVI for the progressive and open approach they took to the evaluation of Bexsero. By publishing an interim statement and accepting subsequent input from various stakeholders, they were able to finally recommend the introduction of the Bexsero vaccine. This greater transparency at every stage of the process is 
warranted and should be a model for vaccine evaluation internationally.

Meningococcal disease is devastatinga disease that every family doctor, emergency department doctor and paediatrician who encounters a febrile child dreads to miss. The 2010 NICE guidelines for its management described it as "...the leading infectious cause of death in early childhood, making its control a priority". ${ }^{18}$ Yet, JCVI found it difficult to recommend the introduction of Bexsero, not because of safety concerns, though there are some, or because of concerns regarding vaccine efficacy. JCVI struggled to support Bexsero's introduction primarily for financial reasons as a result of the economic model used. No modern health system can afford to use a speculative economic model that inhibits introduction of a potentially effective disease prevention strategy. This is especially so for the UK, a country with one of the highest childhood mortality rates in Europe. ${ }^{19}$

Twitter Follow Avishay Sarfatti at @dfg

Contributors All authors contributed to writing and editing the manuscript.

Competing interests $\mathrm{SN}$ has received an educational grant and acted as a consultant for Novartis Vaccines. FM-T has received funding for research and consultancy from Novartis and manufacturers of other meningococcal vaccine. AS has no competing interests to report.

Provenance and peer review Commissioned; externally peer reviewed.

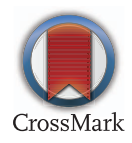

To cite Sarfatti A, Martinón-Torres F, Nadel S. Arch Dis Child 2015;100:514-516.

Received 18 March 2014

Revised 4 January 2015

Accepted 20 January 2015

Published Online First 10 February 2015

Arch Dis Child 2015;100:514-516.

doi:10.1136/archdischild-2014-306030

\section{REFERENCES}

1 European Medicines Agency. Committee for Medicinal Products for Human Use (CHMP). EMA/ CHMP/669278/2012; 15 November 2012.

2 Meningococcal B vaccine: JCVI position statement. Published 21/03/2014. https://www.gov.uk/ government/publications/

meningococcal-b-vaccine-jcvi-position-statement

3 Hall AJ. The United Kingdom Joint Committee on Vaccination and Immunisation. Vaccine 2010;28 (Suppl 1):A54-7.

4 Brysona M, Duclosb P, Jollya A, et al. A global look at national Immunization Technical Advisory Groups. Vaccine 2010;28(Suppl 1):A13-17.

5 Christensen $\mathrm{H}$, Hickman M, Edmunds WJ, et al. Introducing vaccination against serogroup B meningococcal disease: an economic and mathematical modelling study of potential impact. Vaccine 2013;31:2638-46.

6 Joint Committee on Vaccination and Immunisation. JCVI interim position statement on the use of Bexsero meningococcal $B$ vaccine in the UK. Department of Health, 2013. www.gov.uk/ government/publications/jcvi-interim-positionstatement-on-the-use-of-bexseromeningococcal-bvaccine-in-the-uk

7 Christensen $\mathrm{H}$, Trotter $\mathrm{CL}$, Hickman M, et al. Re-evaluating cost effectiveness of universal meningitis vaccination (Bexsero) in England: modelling study. BMJ 2014;349:g5725.

8 Campbell H, Borrow R, Salisbury D, et al. Meningococcal $C$ conjugate vaccine: the experience in England and Wales. Vaccine 2009;27(Suppl 2):B20-9.

9 Ray GT, Whitney CG, Fireman BH, et al. Cost-effectiveness of pneumococcal conjugate vaccine evidence from the first 5 years of use in the
United States incorporating herd effects. Pediatr Infect Dis J 2006;25:494-501.

10 Pouwels KB, Hak E, van der Ende $A$, et al. Cost-effectiveness of vaccination against meningococcal B among Dutch infants: crucial impact of changes in incidence. Hum Vaccin Immunother 2013;9:1129-38.

11 Tu HA, Deeks SL, Morris SK, et al. Economic evaluation of meningococcal serogroup B childhood vaccination in Ontario, Canada. Vaccine 2014;32:5436-46.

12 Campbell $H$, Andrews N, Borrow R, et al. Updated post-licensure surveillance of the meningococcal $C$ conjugate vaccine in England and Wales: effectiveness, validation of serological correlates of protection, and modeling predictions of the duration of herd immunity. Clin Vaccine Immunol 2010;17:840-7.

13 Trotter CL, Edmunds WJ. Reassessing the cost-effectiveness of meningococcal serogroup $\mathrm{C}$ conjugate (MCC) vaccines using a transmission dynamic model. Med Decis Making 2006;26:38-47.

14 Wright C, Wordsworth R, Glennie L. Counting the cost of meningococcal disease: scenarios of severe meningitis and septicemia. Paediatr Drugs 2013;15:49-58.

15 Grimwood $\mathrm{K}$, Anderson P, Anderson V, et al. Twelve year outcomes following bacterial meningitis: further evidence for persisting effects. Arch Dis Child 2000;83:111-16.

16 Rappuoli R, Pizza M, Del Giudice G, et al. Vaccines, new opportunities for a new society. Proc Natl Acad Sci USA 2014;111:12288-93.

17 Joint Committee on Vaccination and Immunisation, Minute of the meeting on 4 June 2014. https://app. box.com/s/iddfb4ppwkmtjusir2tc/1/2199012147/ 19052160649/1

18 NICE clinical guideline 102. Bacterial meningitis and meningococcal septicaemia, Management of bacterial meningitis and meningococcal septicaemia in children and young people younger than 16 years in primary and secondary care. Issued: June 2010.

19 Wolfe I, Thompson M, Gill P, et al. Health services for children in Western Europe. Lancet 2013;381:1224-34. 